\title{
Transformation of double-hit large B-cell lymphoma into B-lymphoblastic lymphoma: a case report
}

\author{
Alexey Glazyrin • Vonda Douglas-Nikitin • \\ James Huang • Mark Micale
}

Received: 14 June 2011 / Accepted: 1 September 2011 /Published online: 29 September 2011

(C) Springer-Verlag 2011

\begin{abstract}
Double-hit lymphomas (DHLs) are highly aggressive B-cell neoplasms that often demonstrate bone marrow and CNS involvement, elevated LDH, and a combination of two cytogenetic events - one involving a rearrangement of the BCL2 gene and the other involving a breakpoint which affects the MYC/8q24 locus, often as the result of a translocation involving immunoglobulin genes IGH, IGK, or IGL. As no effective treatment regimen has yet been defined for such malignancies, DHLs have a poor prognostic outcome. In rare cases, DHLs have demonstrated features consistent with a B-cell lymphoblastic lymphoma. We describe a 55-year-old male who initially presented in March 2010 with significant ascites and extensive lymphadenopathy. A diagnosis of B-cell lymphoma, unclassifiable with features intermediate between diffuse large B-cell lymphoma and Burkitt lymphoma, was made in both peritoneal fluid and bone marrow. The malignant cells expressed CD10, CD19, CD20, CD45 (bright), CD79b, and monotypic kappa light chain, but were negative for TdT.
\end{abstract}

\footnotetext{
A. Glazyrin · V. Douglas-Nikitin · J. Huang $\cdot$ M. Micale

Beaumont Health System, Beaumont Laboratory,

Department of Clinical Pathology, Beaumont Hospital-Royal Oak,

3601 W. Thirteen Mile Road Rd,

Royal Oak, MI 48073, USA

V. Douglas-Nikitin $\cdot$ J. Huang $\cdot$ M. Micale

Department of Pathology and Laboratory Medicine,

Oakland University William Beaumont School of Medicine,

Rochester, MI, USA

M. Micale $(\bowtie)$

Beaumont Health System, Beaumont Laboratory,

Clinical Cytogenomics Laboratory,

3601 W. Thirteen Mile Road Rd,

Royal Oak, MI 48073, USA

e-mail: mmicale@beaumont.edu
}

Both IGH/BCL2 and IGL/MYC gene rearrangements were identified by FISH. Following chemotherapy, the patient remained in morphologic and cytogenetic remission for approximately 4 months. At relapse, a precursor B-cell lymphoma was detected in the diaphragm, pleural wall, and pleural effusion. The malignant cells were TdT positive and were negative for $\mathrm{CD} 20$ and immunoglobulin light chains. Both IGH/BCL2 and IGL/MYC gene rearrangements remained detectable, and a highly complex karyotype was identified in the pleural biopsy. The patient died in November 2010. To our knowledge, this is the first case with clinical and morphological changes documented during disease progression from a diffuse large B-cell lymphoma to a B-cell lymphoblastic lymphoma, both of which possessed IGH/BCL2 and IGL/MYC gene rearrangements.

Keywords B-cell lymphoma · Double-hit lymphomas . BCL2 gene

\section{Introduction}

Double-hit lymphomas (DHLs) are highly aggressive B-cell neoplasms that often demonstrate bone marrow and CNS involvement as well as elevated LDH. As such, they are associated with a poor outcome. These lymphomas are defined by a combination of two cytogenetic events - one involving a rearrangement of the $B C L 2$ gene usually as a result of the $t(14 ; 18)(\mathrm{q} 32 \mathrm{q} 21)$ and the other involving a breakpoint which affects the $M Y C / 8 \mathrm{q} 24$ locus, often partnering in a translocation with various immunoglobulin genes including immunoglobulin heavy chain $(\operatorname{IgH})$, immunoglobulin kappa light chain $(I g K)$, and immunoglobulin lambda light chain $(\operatorname{Ig} L)[1,2]$. The highly aggressive nature of this malignancy occurs both through protection 
from apoptosis by upregulation of the $B C L 2$ antiapoptotic protein due to juxtaposition of the $B C L 2$ gene with $\operatorname{IgH}$, $\operatorname{IgK}$, or $\operatorname{Ig} L$ genes, and by acquiring the ability to proliferate more rapidly through upregulation of MYC [3]. These molecular events predict a very grim prognosis with almost universally fatal outcome $[1,2]$.

No established treatment protocol for DHLs currently exists, partly due to the rarity of diagnosing such lymphomas; however, classification of such cases into the current WHO 2008 intermediate category "lymphoma unclassifiable with features intermediate between diffuse large B-cell lymphoma and Burkitt lymphoma" should result in a more accurate molecular characterization of such malignancies, hopefully leading to an established and effective treatment [4]. In addition, it has been advocated that DHLs actually warrant a separate categorization in future classification schemes due to their clinical and genetic features distinguishing them from other high-grade lymphomas.

Over 300 cases of DHL are described in the literature [1]. Most of these were reported as mature B-cell lymphomas with morphologic and immunophenotypic features suggestive of Burkitt lymphoma (BL), diffuse large B-cell lymphoma (DLBCL), or lymphoma with features intermediate between BL and DLBCL. In even rarer cases, when a DHL demonstrated immature characteristics (TdT positive, CD20 and immunoglobulin light chain negative), they have been classified as B-lymphoblastic lymphoma/leukemia $[2,5]$; however, others refer to classification of such dedifferentiated lymphomas as controversial [6]. Approximately 20 such cases have been described [7-9]. Furthermore, in a recent study examining the clinical and pathological features of 20 DHLs, only one case demonstrated features suggestive of B-lymphoblastic lymphoma [2].

We report an unusual case of DHL with rapid immunophenotypic transformation from mature B-cell lymphoma to a precursor B-cell lymphoma. Such cases of dedifferentiated DHL, B-lymphoblastic type, may behave differently and require a more aggressive treatment regimen than typical DHLs.

\section{Clinical course}

A 55-year-old male was in his usual state of health until March 2010 when he noted early satiety, abdominal distention, and increased abdominal girth. Upon admission, his $\mathrm{CBC}$ was unremarkable except for mild lymphocytosis and mild thrombocytopenia. His LDH was $856 \mathrm{U} / \mathrm{L}$ (normal range, 100-238 u/L), and uric acid was increased. A CT scan revealed intraabdominal, superior mediastinal, and pericardial lymphadenopathy. A large amount of ascites was also noted. Paracentesis removed $7 \mathrm{~L}$ of fluid. Cytologic examination of this peritoneal fluid demonstrated a diffuse large B-cell lymphoma positive for both $I G H / B C L 2$ and $I G L / M Y C$ gene rearrangements by fluorescence in situ hybridization (FISH). The staging bone marrow biopsy revealed extensive lymphoma involvement. Conventional cytogenetic analysis was not performed on the staging bone marrow specimen due to a dry tap.

The patient was treated with six cycles of R-CHOP (cyclophosphamide, doxorubicin, vincristine, prednisone, and rutiximab) and responded favorably with resolution of gastrohepatic, peripancreatic, and aortocaval lymphadenopathy, as well as resolution of the ascites. Some nodular thickening of the anterior aspect of the peritoneum was, however, noted by a CT scan. A PET scan confirmed significant overall improvement; however, two areas of abnormal FDG activity within the left mesentery were concerning for residual disease. A restaging bone marrow biopsy was negative for disease.

In August, repeated imaging studies (both PET and CT) revealed mild interval enlargement of several mesenteric lymph nodes. At the same time, the patient developed a diaphragm mass as well as massive pleural effusion. Both diaphragm and pleural biopsies, as well as pleural fluid, demonstrated a morphologically similar lymphoma with a precursor B-cell immunophenotype, consistent with lymphoblastic transformation. The patient did not respond to additional chemotherapy (RICE regimen) and supportive therapy. He later developed sepsis with acute respiratory failure and expired in November 2010.

\section{Materials and methods}

Morphologic analysis and immunohistochemistry

Pleural and abdominal fluid cells were concentrated by centrifugation according to standard laboratory protocol. Concentrated cellular material and a needle core biopsy of the diaphragm and pleura were fixed in buffered formalin, paraffin embedded, and stained with hematoxylin and eosin. Immunohistochemical studies included CD3, CD5, CD10 (Thermo Scientific), CD79a, Ki-67, TdT, BCL2, BCL6 (DAKO, Carpinteria, CA). Wright stains were performed for bone marrow (BM) aspirate, peripheral blood, pleural fluid, and ascites for morphological evaluation. The BM biopsy specimens were decalcified in rapid decalcification solution for $1 \mathrm{~h}$. The BM core biopsies were sectioned and stained with hematoxylin-eosin.

\section{Flow cytometry}

The original diagnostic peritoneal fluid as well as subsequent BM aspirates, diaphragmatic biopsies, and pleural fluid were submitted for flow cytometric immunophenotyp- 
ing. A single-cell suspension was analyzed on an FC500 flow cytometer (Beckman-Coulter, Brea, CA) with standard techniques for 5-color analysis utilizing the following commercially available monoclonal antibodies: CD2, CD3, CD4, CD5, CD7, CD8, CD10, CD14, CD16, CD19, CD20, CD22, CD23, CD33, CD34, CD38, CD45, CD56, CD57, CD79b, CD117, kappa and lambda immunoglobulin light chains, and intracellular kappa, lambda and bcl-2 (BD Biosciences, San Jose, CA). Additionally, staining for surface kappa and lambda chains using commercially available polyclonal antibodies (BD Biosciences, San Jose, CA) was performed.

\section{Cytogenetic analysis}

Both peritoneal fluid and bone marrow biopsies were submitted throughout the patient's illness for conventional cytogenetic analysis and FISH. Unstimulated bone marrow cultures were incubated for $24 \mathrm{~h}$ and harvested according to standard protocols. GTL-banded metaphase cells were analyzed and described according to the International System for Human Cytogenetic Nomenclature (2009). FISH was performed using standard laboratory protocols. The presence of $I G H / B C L 2$ gene rearrangement was assessed utilizing the LSI IGH/BCL2 Dual Color, Dual Fusion Translocation Probe while $I G H / M Y C$ rearrangement was identified utilizing the LSI IGH/MYC, CEP 8 Tri Color Dual Fusion Translocation DNA probe (Abbott Molecular, Inc., Des Plains, IL). MYC gene rearrangement was assessed with the LSI MYC gene rearrangement probe (Abbott Molecular, Inc., Des Plains, IL), while rearrangement of the Ig kappa and Ig lambda genes was determined utilizing the $I G K(2 \mathrm{p} 12)$ and $I G L(22 \mathrm{q} 11.2)$ rearrangement probes (DakoCytomation, Denmark), respectively.

\section{Results}

Morphologic examination of the diagnostic peritoneal fluid specimen reveals a double-hit diffuse large B-cell lymphoma

Cytological features of lymphoma cells from the diagnostic peritoneal fluid specimen collected in March 2010 are depicted in Fig. 1a. The lymphoma cells were predominately large and polymorphic with cytoplasmic vacuoles. Some cells showed degeneration and apoptosis. Immunohistochemical analysis performed on the cell block of the peritoneal fluid demonstrated that tumor cells were strongly positive for CD20, BCL2, and CD10, and variably positive for BCL6. TdT was not detected (Fig. 1b). Approximately $80 \%$ of the cells were Ki-67 positive. This fluid also demonstrated $M Y C$ and $I G L$ gene rearrangements (Fig. 1c and d), as well as IGH/BCL2 gene rearrangement by FISH.
Flow cytometric analysis of the diagnostic peritoneal fluid revealed medium-sized to large monotypic $B$ cells, expressing CD10, CD19, CD20, CD45 (bright), CD79b, and monotypic kappa light chain. These monoclonal $\mathrm{B}$ cells were negative for CD5 and CD23. Representative histograms are shown in Fig. $2 a$ and $b$. The differential diagnosis included diffuse large B-cell lymphoma, Burkitt lymphoma, and a grade 3 follicular lymphoma.

A simultaneous staging bone marrow biopsy revealed extensive involvement by a lymphoid infiltrate with confluent areas of large lymphoid cells, areas admixed with smaller lymphoid cells, and areas occupied by a small-cell lymphoma (Fig. 2c). Immunohistochemical analysis of the bone marrow infiltrate demonstrated an immunophenotype similar to that identified in peritoneal fluid. Tumor cells were CD20 positive (Fig. 2d) and Ki-67 positive in approximately 50\% of lymphoma cells. TdT was negative. Positive immunohistochemical staining of the $\mathrm{B}$ cells with $\mathrm{CD} 10$ and $B C L 2$ suggests the bone marrow infiltrate may have arisen from a follicular lymphoma. However, FISH analysis of the bone biopsy also revealed both $I G L / M Y C$ and $I G H / B C L 2$ gene rearrangements. Concurrent analysis of cerebrospinal fluid failed to reveal central nervous system involvement by lymphoma.

Transformation of the original double-hit B-cell lymphoma to a precursor B-cell immunophenotype

A bone marrow biopsy performed in August 2010 (approximately 6 months after the original diagnosis and following chemotherapy and Neupogen treatment) demonstrated a hypercellular bone marrow with adequate trilineage hematopoiesis without evidence of bone marrow involvement by residual lymphoma. This morphological impression was confirmed by flow cytometry.

The diaphragm mass biopsy performed in August 2010 revealed lymphoma relapse (Fig. 3). Extensive infiltration of polymorphic large lymphoma cells was seen within the skeletal muscle fibers. By flow cytometry, the cells continued to demonstrate positivity for CD10, CD19, and CD45, but became negative for $\mathrm{CD} 20$ as well as for surface and cytoplasmic immunoglobulin light chains. A small percentage of lymphoma cells were positive for TdT.

The pleural biopsy performed in October 2010 showed diffuse proliferation of lymphoma cells (Fig. 4) positive for CD79a, but negative for CD20. TdT positivity was significantly increased compared with the previous diaphragm biopsy. The Ki-67 proliferation index ranged from $60 \%$ to $80 \%$. The pleural fluid collected simultaneously revealed morphologically similar lymphoma cells comparable with those identified in the diagnostic ascites fluid specimen. Flow cytometry of the pleural fluid revealed an immunophenotype similar to that of the diaphragmatic biopsy, but different from that at initial presentation. Although CD45 
Fig. 1 Histological and molecular cytogenetic evaluation of diagnostic peritoneal fluid (March 2010). Significant cytoplasmic vacuolization was noted in the majority of tumor cells (a) (magnification, $\times 350$ ). No significant positive TdT staining was detected by immunohistochemistry on the peritoneal fluid cell block (b). Both $\operatorname{Ig} L$ gene rearrangement (c) and $M Y C$ gene rearrangement $(\mathbf{d})$ were detected by FISH
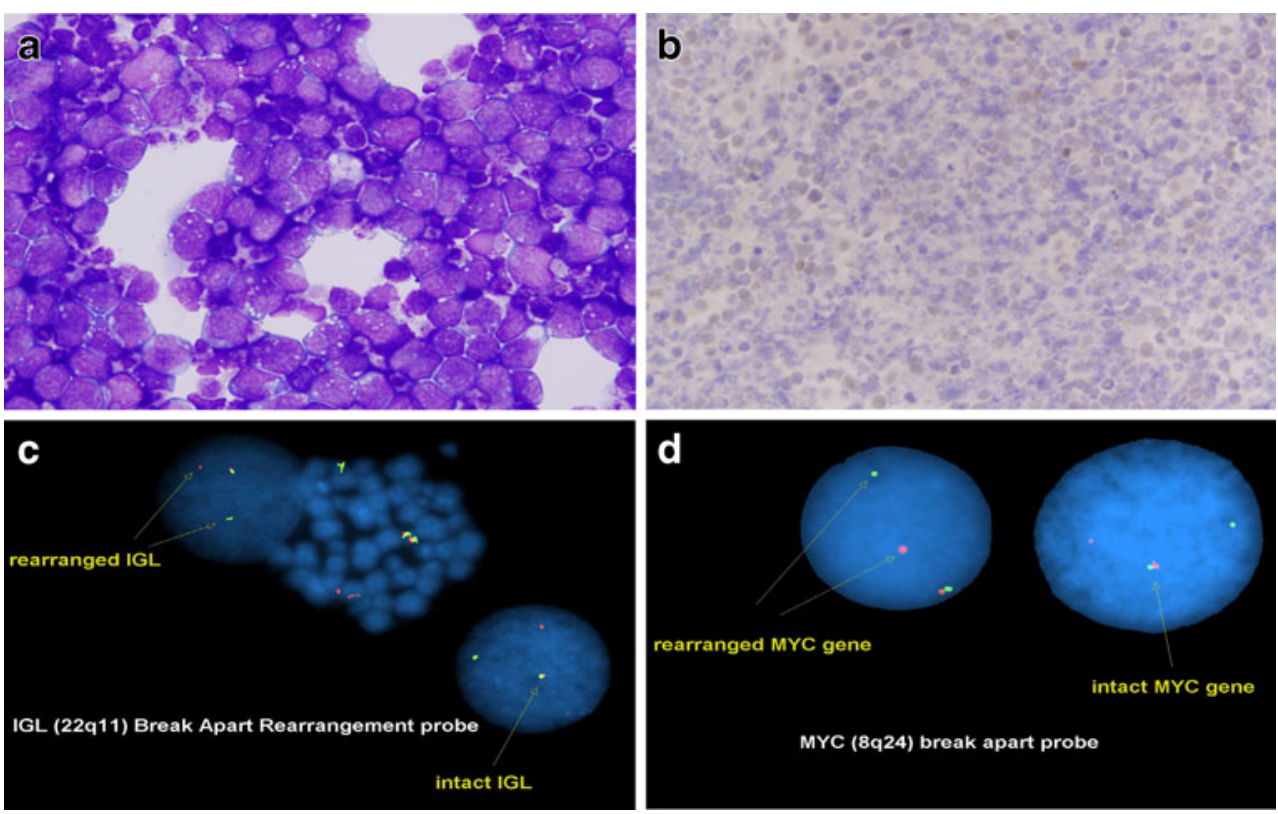

remained bright, CD20 and light chains were no longer detectable. A concurrent bone marrow biopsy was negative for tumor involvement morphologically, immunophenotypically, and by cytogenetic analysis.

Cytogenetic analysis of the pleural biopsy demonstrated the complex karyotype: 45-49, XY,der(1)t(1;1)(p36.3;q22), del(6) (q21),t(8;22)(q24;q11),t(14;18)(q32;q21),+1-3mar [cp20]. FISH analysis identified both $I G H / B C L 2$ and $I G L / M Y C$ gene rearrangements, each in 200 nuclei. In total, these FISH studies were described karyotypically as: nuc ish $(I G K \times 2)$ [200],(MYCx2)(5' MYC sep 3'MYCx1)[198/200],(D8Z2x2,
$M Y C \times 2, I G H \times 3)[195 / 200],(I G H, B C L 2) \times 3(I G H$ con $B C L 2 \times 2)[200],(I G L \times 2)\left(5^{\prime} I G L\right.$ sep $\left.3^{\prime} I G L \times 1\right)[200]$.

\section{Discussion}

To our knowledge, this is the first documented case of a double-hit large B-cell lymphoma with rapid transformation from a mature large B-cell lymphoma to a precursor B-cell lymphoma. Clearly, the lymphoma at initial presentation had an immunophenotype of mature B cells supported by the
Fig. 2 Histological and flow cytometric evaluation of diagnostic peritoneal fluid and initial bone marrow biopsy (March 2010). Flow cytometric analysis of peritoneal fluid cells revealed tumor cells to be monotypic with kappa light chain restriction (a) and positive for CD20 and CD10 (b). Bone marrow biopsy showed hypercellular bone marrow with extensive infiltrate of a mixture of small and large lymphocytes (c) (magnification, $\times 100$ ). The majority of lymphoid cell were positive for CD20 (d) (magnification, $\times 100$ ) a
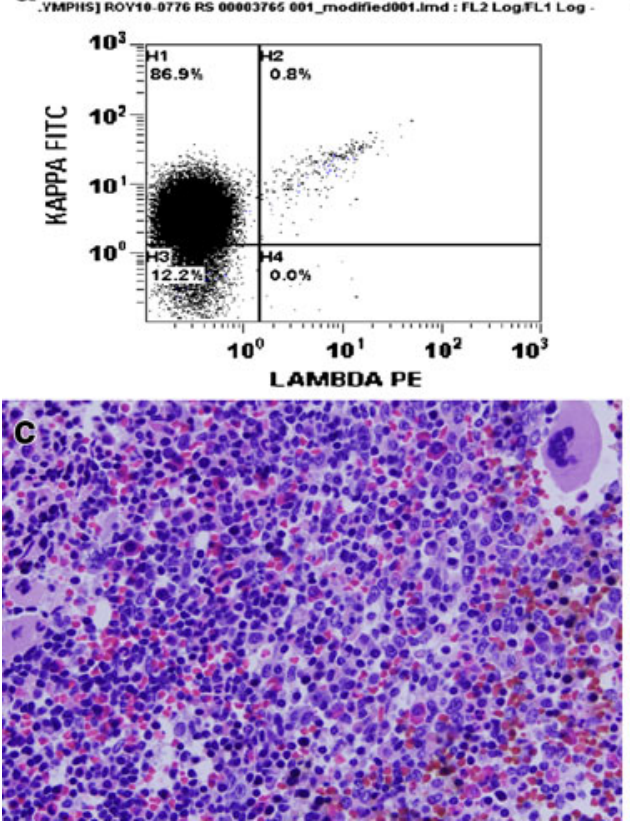

b
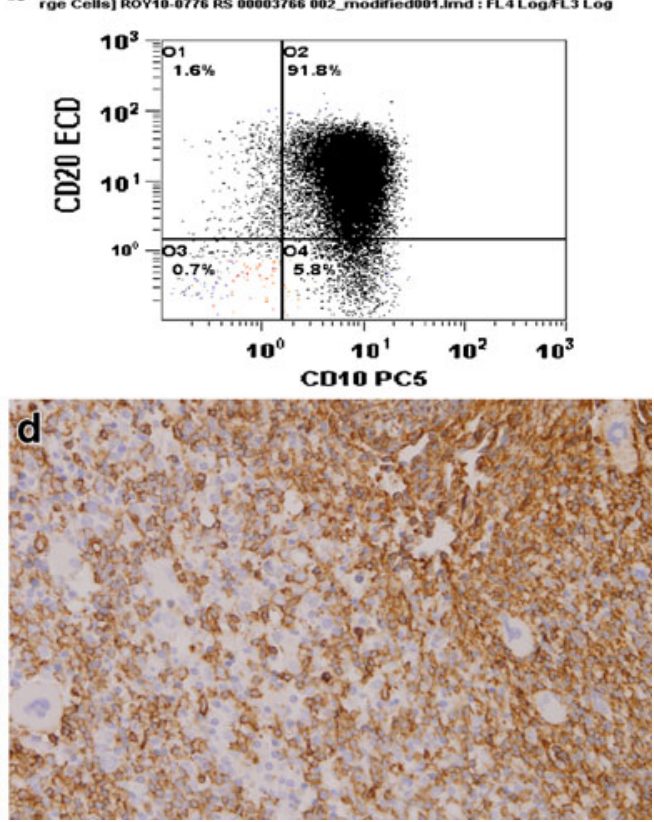
Fig. 3 Histological, immunohistochemical, and flow cytometric studies of diaphragm biopsy in August 2010. Striated muscle cells of the diaphragm showed extensive infiltration of large lymphoma cells (a). Some lymphoma cells expressed TdT (b) (magnification, $\times 200$ ). Flow cytometry demonstrated lymphoma cells to be negative for CD20 and kappa light chain (c) but positive for CD19 and CD10 (d)
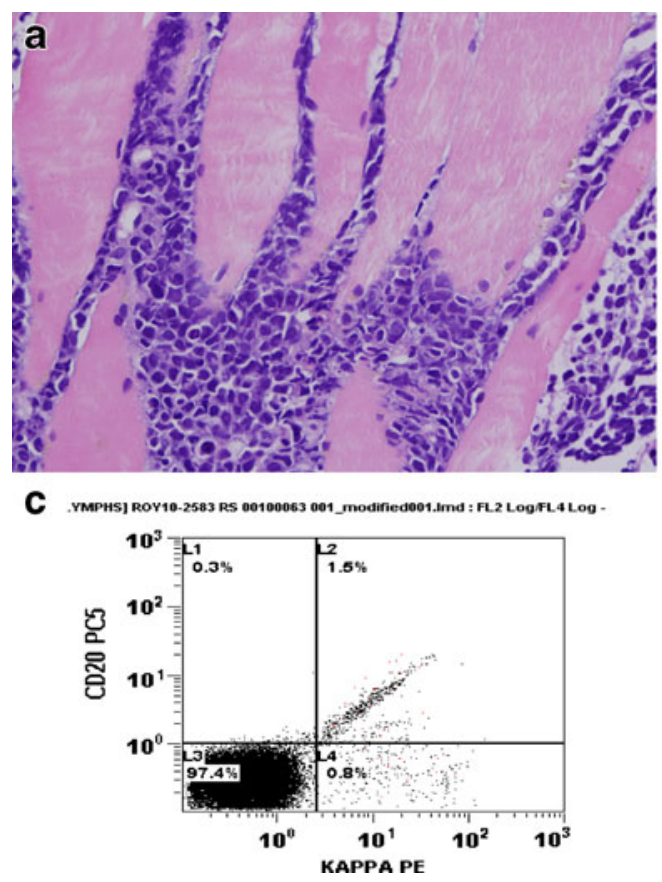

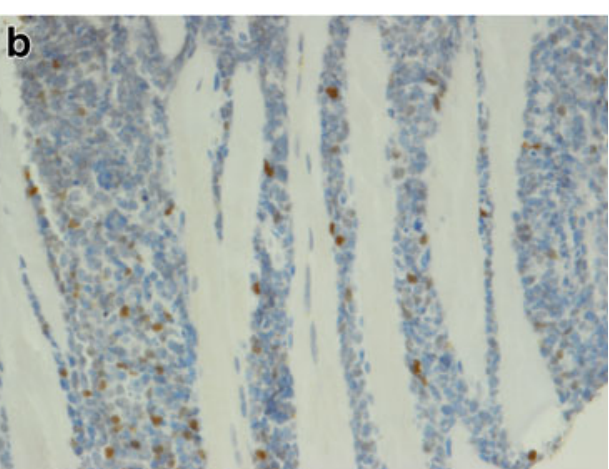

d ,

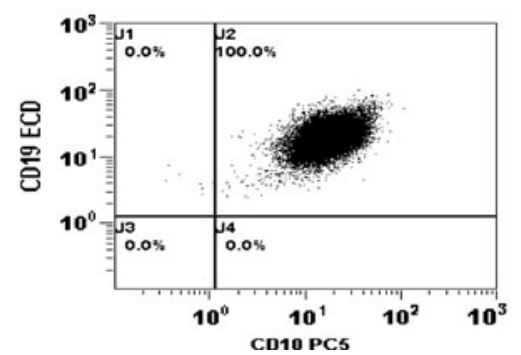

expression of CD45 (bright), CD20, and surface light chain restriction in the absence of expression of CD34 and TdT. The lymphoma could best be classified as "lymphoma, unclassifiable, with features intermediate between diffuse large B-cell lymphoma and Burkitt lymphoma" based on the current WHO classification scheme. Given the aggressive clinical features and high LDH level, treatment was initiated without obtaining any additional biopsies of potentially involved abdominal lymph nodes.

The standard treatment protocol (CHOP-R) for DLBCL was used and effectively cleared the bone marrow of malignancy; however, clinical remission was short with relapse occurring within 4 months. The recurrent lymphoma had a precursor B-cell immunophenotype consistent with transformation (or dedifferentiation) characterized by loss of both CD20 and immunoglobulin light chains, as well as acquisition of TdT. Although the history of recent rutuximab therapy may account for the loss of CD20, it typically does affect immunoglobulin light chain expression. TdT-positive lymphoma cells initially emerged in the diaphragmatic biopsy and become more numerous in the subsequent pleural biopsy. To rule out the possibility of technical variability of immunohis-
Fig. 4 Confirmation of a persistent B cell phenotype in the pleural biopsy in October 2010. The pleural biopsy showed diffuse proliferation of large lymphoma cells (a) (magnification, $\times 200$ ). TdTpositive lymphoma cells were increased (b). Lymphoma cells showed variable expression of $\mathrm{Ki}-67$ (c), but relatively uniform expression of CD79a (d)
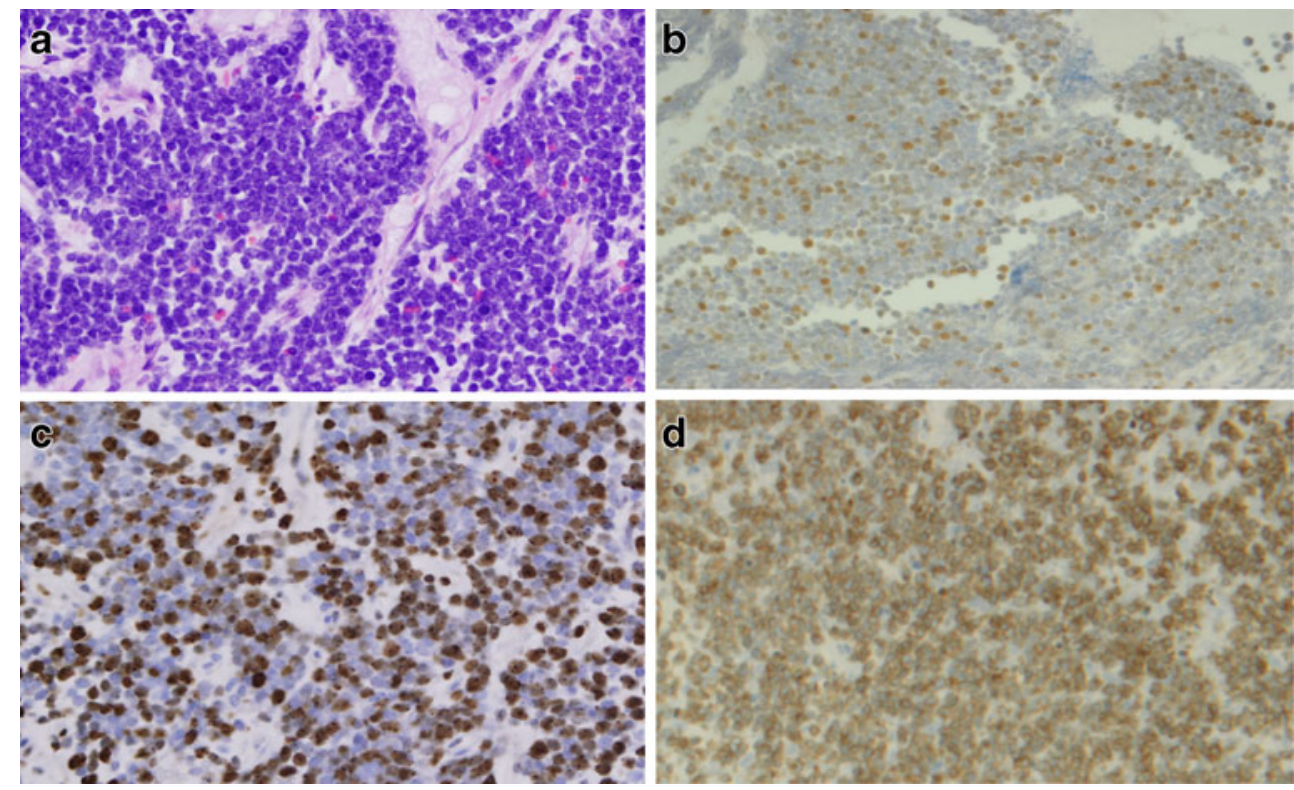
tochemical staining as a cause for the difference of TdTpositive cell percentages between the diaphragm and pleural biopsies, additional tissue blocks from both diaphragm and pleural biopsies were stained with TdT, and confirmed pleural biopsy had more TdT-positive cells than diaphragm biopsy. The positive control tissues used for both specimens were carefully compared, and they showed consistent quality of staining. Therefore, we believe such an increase in TdTpositive cells suggests ongoing transformation or dedifferentiation during disease progression. While re-expression of TdT can also occur as a result of B cells accumulating somatic hypermutations, our case presented additional findings which support dedifferentiation.

An alternative explanation for these findings may be the selective regression of a DLBCL lymphoma subclone originating from a primary double-hit follicular lymphoma. In this model, the other component, which in this case would be an occult parallel lymphoblastic lymphoma subclone, could have subsequently predominated, thereby giving the morphological impression of dedifferentiation of a primary B-cell lymphoma. In theory, regression of one of these phenotypically divergent (bilineal) subclones could have been the result of selective pressure of the patient's CHOP-R therapy.

Double-hit lymphomas, including the present case, seem to acquire their characteristic genetic rearrangements in a progressive step-wise fashion, with the first hit involving $B C L 2$ gene rearrangement and the second hit involving a breakpoint at $8 \mathrm{q} 24 / M Y C$ often as part of a rearrangement with either $I G L$ or $I G K$ genes associated with the $t(8: 22)$ (q24;q11) or $t(2 ; 8)(\mathrm{p} 12 ; \mathrm{q} 24)$, respectively. Some have speculated that this association specifically with $I G L$ and $I G K$ genes may occur because an already rearranged $I G H$ gene is not available to participate in a reciprocal translocation with $M Y C$, as occurs in classical Burkitt lymphoma $[1,2,8]$. We have, however, recently identified a case of DHL with both $I G H / B C L 2$ and $I G H / M Y C$ rearrangements, so this dual fusion combination certainly does occur.

Current practice dictates that any lymphoma suspected of being a double-hit lymphoma (especially with a high Ki-67 proliferation index) should be analyzed for both $M Y C$ and BCL2 gene rearrangements. Double-hit lymphomas typically have a Ki-67 proliferation index $>80 \%$; however, a lower Ki-67 index has been observed in such lymphomas, as in the present case. Therefore, a decision to submit tissue for gene rearrangement FISH studies should not entirely be based on the Ki-67 index, as it can be potentially affected by tumor cell degeneration and ongoing apoptosis. Similar to previously reported cases, our patient demonstrated a very high LDH level (more than three times the upper limit of normal) at presentation. This finding is considered a relatively specific marker for distinguishing DHL patients from those with either Burkitt's lymphoma or
DLBCL (NOS) $[1,2]$. If the clinical presentation and LDH level are suggestive of a high-grade process, FISH analysis for both $M Y C$ and $B C L 2$ gene rearrangements should be performed as early as possible in order to make a timely and definitive diagnosis of double-hit B-cell lymphoma.

Transformation from low-grade to high-grade is often a part of the natural history of B-cell lymphoma. Transformation from follicular lymphoma into a double-hit large B-cell lymphoma as well as directly to lymphoblastic lymphoma has been occasionally reported [7, 10]. Our patient did not have a documented history of follicular lymphoma. The initial bone marrow biopsy showed lymphoma cells of admixed large and small cells, raising a possibility that the patient may have a concurrent follicular lymphoma; however, FISH analysis of this bone marrow biopsy showed both IGH/BCL2 and IGL/MYC gene rearrangements arguing against the presence of a concurrent follicular lymphoma. A preceding follicular lymphoma could not, however, be entirely ruled out because follicular lymphoma may be asymptomatic and not diagnosed. Controversy exists as to whether double-hit B-lymphoblastic lymphoma can appear de novo or whether it represents lymphoblastic transformation from a preceding B-cell lymphoma $[8,11]$. Our case suggests that mature double-hit large B-cell lymphomas may dedifferentiate or transform into lymphoblastic lymphoma, at least in some cases, and that this can happen relatively quickly.

In summary, we describe a case of DHL lymphoma which demonstrated clinical and biological progression from mature B-cell lymphoma, unclassifiable with features intermediate between diffuse large B-cell lymphoma and Burkitt lymphoma, to a B-cell lymphoblastic lymphoma. To our knowledge this is the first case described with clinical and morphological changes documented during disease progression. Correct treatment strategy for this highly aggressive disease with a grim prognosis remains to be elucidated, but separate subcategorization of this entity in future classification schemes as well as registration of all known cases may bring us closer to establishing an effective treatment.

Conflict of interest The authors declare that they have no conflict of interest.

\section{References}

1. Aukema SM, Siebert R, Schuuring E, van Imhoff GW, KluinNelemans HC, Boerma EJ, Kluin PM (2011) Double-hit B-cell lymphomas. Blood 117(8):2319-2331

2. Snuderl M, Koman OK, Chen YB, Hsu JJ, Ackerman AM, Dal CP, Ferry JA, Harris NL, Hasserjian PR, Zukerberg LR, Abramson JS, Hochberg EP, Lee H, Lee AI, Toomey CE, Sohani AR (2010) B-cell lymphomas with concurrent IGHBCL2 and MYC rearrangements are aggressive neoplasms 
with clinical and pathologic features distinct from Burkitt lymphoma and diffuse large B-cell lymphoma. Am J Surg Pathol 34(3):327-340

3. Voorhees PM, Carder KA, Smith SV, Ayscue LH, Rao KW, Dunphy CH (2004) Follicular lymphoma with a Burkitt translocation-predictor of an aggressive clinical course: a case report and review of the literature. Arch Pathol Lab Med 128:210-213

4. Swerdlow SS, Campo E, Harris NL, Jaffe ES, Pileri SA, Stein H, Thiele J, Vardiman JW (eds) (2008) WHO classification of tumours of haematopoietic and lymphoid tissues. IARC Press, Lyon

5. Hasserjian RP, German Ott, Elenitoba-Johnson KSJ, Balague-Ponz O, de Jong D, de Leval L (2009) Commentary on the WHO classification of tumors of lymphoid tissues 2008: "Gray zone" lymphomas overlapping with Burkitt lymphoma or classical Hodgkin lymphoma. J Hematopathology 2(2):89-95

6. de Jong D (2009) Novel lymphoid neoplasms-the borderland between diffuse large B-cell lymphoma and Burkitt's lymphoma. Haematologica 94:894-896
7. Young KH, Xie Q, Zhou G, Eickhoff JC, Sanger WG, Aoun P, Chan WC (2008) Transformation of follicular lymphoma to precursor B-cell lymphoblastic lymphoma with c-myc gene rearrangement as a critical event. Am J Clin Pathol 129:157-166

8. Brito-Babapulle V, Crawford A, Khokhar T, Laffan M, Matutes E, Fairhead S, Catovsky D (1991) Translocations $t(14 ; 18)$ and $t$ $(8 ; 14)$ with rearranged bcl-2 and c-myc in a case presenting as B-ALL (L3). Leukemia 5:83-87

9. D'Achille P, Seymour JF, Campbell LJ (2006) Translocation (14;18) (q32;q21) in acute lymphoblastic leukemia: a study of 12 cases and review of the literature. Cancer Genet Cytogenet 171:52-56

10. Johnson NA, Savage KS, Ludkovski O, Ben-Neriah S, Woods R, Steidl KS, Dyer MJS, Siebert R, Kuruvilla J, Klasa R, Connors JM, Gascoyne RD, Horsman DE (2009) Lymphomas with concurrent $B C L 2$ and MYC translocations: the critical factors associated with survival. Blood 114(11):2273-2279

11. Gurbaxani S, Anastasi J, Hyjek E (2009) Diffuse large B-cell lymphoma - more than a diffuse collection of large B cells. An entity in search of meaningful classification. Arch Pathol Lab Med $133: 1121-1134$ 